\title{
NONEXISTENCE OF GLOBAL SOLUTIONS OF A DELAYED WAVE EQUATION WITH VARIABLE-EXPONENTS
}

\author{
ERHAN PIŞKIN AND HAZAL YÜKSEKKAYA
}

Received 15 October, 2020

\begin{abstract}
This work deals with a Petrovsky equation with delay term and variable exponents. Firstly, we establish the local existence result by the Faedo-Galerkin method. Later, we prove the blow-up of solutions in a finite time. Our results are more general than the earlier results.
\end{abstract}

2010 Mathematics Subject Classification: 35B44; 35L05; 35L55

Keywords: blow up, existence, Petrovsky equation, delay term, variable exponent

\section{INTRODUCTION}

In this work, we study the following Petrovsky equation with variable exponents and delay term

$$
\begin{cases}u_{t t}+\Delta^{2} u-\Delta u_{t}+\mu_{1} u_{t}(x, t)\left|u_{t}\right|^{m(x)-2}(x, t) & \text { in } \Omega \times R^{+}, \\ +\mu_{2} u_{t}(x, t-\tau)\left|u_{t}\right|^{m(x)-2}(x, t-\tau)=b u|u|^{p(x)-2} & \\ u(x, t)=\frac{\partial u(x, t)}{\partial v}=0 & \text { in } \partial \Omega \times[0, \infty), \\ u(x, 0)=u_{0}(x), u_{t}(x, 0)=u_{1}(x) & \text { in } \Omega, \\ u_{t}(x, t-\tau)=f_{0}(x, t-\tau) & \text { in } \Omega \times(0, \tau),\end{cases}
$$

where $\Omega$ is a bounded domain with smooth boundary $\partial \Omega$ in $R^{n}, n \geq 1$. Here, $\tau>0$ is a time delay term, $b \geq 0$ is a constant, $\mu_{1}$ is a positive constant and $\mu_{2}$ is a real number. The functions $u_{0}, u_{1}, f_{0}$ are the initial data to be specified later.

The variable exponents $p(\cdot)$ and $m(\cdot)$ are given as measurable functions on $\bar{\Omega}$ satisfy:

$$
\left\{\begin{array}{l}
2 \leq m^{-} \leq m(x) \leq m^{+} \leq m^{*} \\
2 \leq p^{-} \leq p(x) \leq p^{+} \leq p^{*}
\end{array}\right.
$$

where

$$
\begin{array}{ll}
m^{-}=e \operatorname{sinf} m(x), & m^{+}=e s s \sup _{x \in \Omega} m(x), \\
p^{-}=e s \operatorname{sinf}_{x \in \Omega} p(x), & p^{+}=e s s \sup _{x \in \Omega} p(x),
\end{array}
$$

(C) 2021 Miskolc University Press 
and

$$
m^{*}, p^{*}=\frac{2(n-2)}{n-4} \quad \text { if } n>4 .
$$

The problems with variable exponents arises in many branches in sciences such as electrorheological fluids, nonlinear elasticity theory and image processing [3, 4, 20 23]. Time delay often appears in many practical problems like thermal, biological, chemical, physical and economic phenomena [7].

There has been published much work concerning the wave equations with variable exponents or time delay. Our goal is to consider the Petrovsky equation both with the delay term $\left(\mu_{2} u_{t}(x, t-\tau)\right)$ and variable exponents which make the problem more interesting than from those concerned in the literature.

$\mathrm{Li}$ et al. [11] considered the Petrovsky equation with strong damping term as follows

$$
u_{t t}+\Delta^{2} u-\Delta u_{t}+\left|u_{t}\right|^{p-2} u_{t}=|u|^{q-2} u .
$$

The authors established the blow up of solutions, existence and decay of the problem (1.3). Then, Polat and Pişkin [19] proved the global existence and decay of solutions of (1.3).

In [12], Messaoudi studied the Petrovsky equation as follows

$$
u_{t t}+\Delta^{2} u+g\left(u_{t}\right)=\beta|u|^{r-1} u,
$$

where $g\left(u_{t}\right)=\alpha\left|u_{t}\right|^{p-1} u_{t}$ and he investigated the blow-up result in finite time for $r>p$. In [25], for when $g\left(u_{t}\right)=\alpha\left|u_{t}\right|^{p-1} u_{t}$, Tsai and Wu looked into that the solution is global for equation (1.4). Moreover, they established the blow-up result in finite time for the nonnegative initial energy.

Messaoudi and Kafini [6] looked into the nonlinear wave equation with variable exponents and delay term as follows

$u_{t t}-\Delta u+\mu_{1} u_{t}(x, t)\left|u_{t}\right|^{m(x)-2}(x, t)+\mu_{2} u_{t}(x, t-\tau)\left|u_{t}\right|^{m(x)-2}(x, t-\tau)=b u|u|^{p(x)-2}$.

They proved the global nonexistence and decay estimates of the equation (1).

In recent years, some other authors investigate hyperbolic type equation with variable exponents (see [8,13,16, 18, 24]).

There is no research, to our best knowledge, about Petrovsky equation with delay term and variable exponents, hence, our paper is generalization of the previous ones. In this paper, our main goal is to study the local existence and blow-up result of Petrovsky equation (1.1) with variable exponents and delay term.

The plan of this paper is as follows. Firstly, in Section 2, the definition of the variable exponent Sobolev and Lebesgue spaces are introduced. In Section 3, we obtain the local existence result. Finally, in Section 4, we prove the blow-up result for negative initial energy. 


\section{PRELIMINARIES}

In this part, we state the results related to Lebesgue $L^{p(\cdot)}(\Omega)$ and Sobolev $W^{1, p(\cdot)}(\Omega)$ spaces with variable exponents (see $\left.[1,2,4,5,10,17]\right)$.

Let $p: \Omega \rightarrow[1, \infty)$ be a measurable function. We define the variable exponent Lebesgue space with a variable exponent $p(\cdot)$ by

$$
L^{p(\cdot)}(\Omega)=\left\{u: \Omega \rightarrow R \text {; measurable in } \Omega: \int_{\Omega}|u|^{p(\cdot)} d x<\infty\right\},
$$

with a Luxemburg-type norm

$$
\|u\|_{p(\cdot)}=\inf \left\{\lambda>0: \int_{\Omega}\left|\frac{u}{\lambda}\right|^{p(x)} d x \leq 1\right\} .
$$

Equipped with this norm, $L^{p(\cdot)}(\Omega)$ is a Banach space (see [4]).

Next, we define the variable-exponent Sobolev space $W^{1, p(\cdot)}(\Omega)$ as following:

$$
W^{1, p(\cdot)}(\Omega)=\left\{u \in L^{p(\cdot)}(\Omega): \nabla u \text { exists and }|\nabla u| \in L^{p(\cdot)}(\Omega)\right\} .
$$

Variable exponent Sobolev space with the norm

$$
\|u\|_{1, p(\cdot)}=\|u\|_{p(\cdot)}+\|\nabla u\|_{p(\cdot)}
$$

is a Banach space. $W_{0}^{1, p(\cdot)}(\Omega)$ is the space which is defined as the closure of $C_{0}^{\infty}(\Omega)$ in $W^{1, p(\cdot)}(\Omega)$. For $u \in W_{0}^{1, p(\cdot)}(\Omega)$, we can define an equivalent norm

$$
\|u\|_{1, p(\cdot)}=\|\nabla u\|_{p(\cdot)} .
$$

The dual of $W_{0}^{1, p(\cdot)}(\Omega)$ is defined as $W_{0}^{-1, p^{\prime}(\cdot)}(\Omega)$, similar to Sobolev spaces, where

$$
\frac{1}{p(\cdot)}+\frac{1}{p^{\prime}(\cdot)}=1 \text {. }
$$

We also assume that:

$$
|p(x)-p(y)| \leq-\frac{A}{\log |x-y|} \quad \text { and } \quad|m(x)-m(y)| \leq-\frac{B}{\log |x-y|}
$$

for all $x, y \in \Omega, A, B>0$ and $0<\delta<1$ with $|x-y|<\delta$ (log-Hölder condition).

Lemma 1 ([1] Poincare inequality). Let $\Omega$ be a bounded domain of $R^{n}$ and suppose that $p(\cdot)$ satisfies (2.1). Then,

$$
\|u\|_{p(\cdot)} \leq c\|\nabla u\|_{p(\cdot)} \quad \text { for all } u \in W_{0}^{1, p(\cdot)}(\Omega),
$$

where $c=c\left(p^{-}, p^{+},|\Omega|\right)>0$.

Lemma 2 ([1]). If $p: \bar{\Omega} \rightarrow[1, \infty)$ is continuous,

$$
2 \leq p^{-} \leq p(x) \leq p^{+} \leq \frac{2 n}{n-2}, \quad n \geq 3,
$$


satisfies, then the embedding $H_{0}^{1}(\Omega) \hookrightarrow L^{p(\cdot)}(\Omega)$ is continuous.

Lemma 3 ([1]). If $p^{+}<\infty$ and $p: \Omega \rightarrow[1, \infty)$ is a measurable function, then $C_{0}^{\infty}(\Omega)$ is dense in $L^{p(\cdot)}(\Omega)$.

Lemma 4 ([1] Hölder' inequality). Let $p, q, s \geq 1$ be measurable functions defined on $\Omega$ and

$$
\frac{1}{s(y)}=\frac{1}{p(y)}+\frac{1}{q(y)}, \quad \text { for a.e. } y \in \Omega,
$$

satisfies. If $f \in L^{p(\cdot)}(\Omega)$ and $g \in L^{q(\cdot)}(\Omega)$, then $f g \in L^{s(\cdot)}(\Omega)$ and

$$
\|f g\|_{s(\cdot)} \leq 2\|f\|_{p(\cdot)}\|g\|_{q(\cdot)} \cdot
$$

Lemma 5 ([6, Lemma 2.5] unit ball property). Let $p \geq 1$ be a measurable function on $\Omega$. Then $\|f\|_{p(\cdot)} \leq 1$ if and only if $\rho_{p(\cdot)}(f) \leq 1$, where

$$
\rho_{p(\cdot)}(f)=\int_{\Omega}|f(x)|^{p(x)} d x .
$$

Lemma 6 ([1]). If $p \geq 1$ is a measurable function on $\Omega$, then

$$
\min \left\{\|u\|_{p(\cdot)}^{p^{-}},\|u\|_{p(\cdot)}^{p^{+}}\right\} \leq \rho_{p(\cdot)}(u) \leq \max \left\{\|u\|_{p(\cdot)}^{p^{-}},\|u\|_{p(\cdot)}^{p^{+}}\right\},
$$

for any $u \in L^{p(\cdot)}(\Omega)$ and for a.e. $x \in \Omega$.

Remark 1. Let $c$ be various positive constants which may be different from line to line. Then, we use the embedding

$$
H_{0}^{2}(\Omega) \hookrightarrow H_{0}^{1}(\Omega) \hookrightarrow L^{p}(\Omega)
$$

which satisfies

$$
\|u\|_{p} \leq c\|\nabla u\| \leq c\|\Delta u\|,
$$

where $2 \leq p<\infty(n=1,2), 2 \leq p \leq \frac{2 n}{n-2}(n \geq 3)$.

Moreover

$$
\begin{gathered}
\|u\|_{q} \leq C\|\Delta u\|, \\
q= \begin{cases}\infty & \text { if } n<4, \\
\text { any number in }[1, \infty) & \text { if } n=4, \\
\frac{2(n-2)}{n-4} & \text { if } n>4 .\end{cases}
\end{gathered}
$$




\section{LOCAL EXISTENCE}

In this part, our goal is to prove the local existence result for our main problem (1.1) by using Faedo-Galerkin method. We use similar arguments as in $[14,16]$ to get the result. Firstly, we give the lemma which we need:

Lemma 7 ([9, Lemma 3.1]). (Lemma 3.1 in [9]) Let $x \in \Omega$ and $p(\cdot)$ satisfies

$$
2 \leq p^{-} \leq p(x) \leq p^{+} \leq \infty,
$$

then, $h(s)=b|s|^{p(x)-2} s$ is differentiable function and $\left|h^{\prime}(s)\right|=b|p(x)-1||s|^{p(x)-2}$.

Suppose that $\mu_{1}$ and $\mu_{2}$ satisfy

$$
\left|\mu_{2}\right|<\frac{m^{-}}{m^{+}} \mu_{1},
$$

where $m^{-}=e s \sin f_{x \in \Omega} m(x), m^{+}=e s \sup f_{x \in \Omega} m(x)$. Assume that $\zeta$ is a positive constant such that

$$
\tau\left(m^{+}-1\right) \mu_{2}<\zeta<\tau\left(m^{-} \mu_{1}-\left|\mu_{2}\right|\right) .
$$

Now, similar to [15], we introduce, the new variable

$$
z(x, \rho, t)=u_{t}(x, t-\tau \rho), \quad x \in \Omega, \rho \in(0,1), t>0 .
$$

Hence, problem (1.1) takes the form

$$
\begin{cases}u_{t t}+\Delta^{2} u-\Delta u_{t}+\mu_{1}\left|u_{t}(x, t)\right|^{m(x)-2} u_{t}(x, t) & \text { in } \Omega \times R^{+}, \\ +\mu_{2}|z(x, 1, t)|^{m(x)-2} z(x, 1, t)=b u|u|^{p(x)-2} & \\ \tau z_{t}(x, \rho, t)+z_{\rho}(x, \rho, t)=0 & \text { in } \Omega \times(0,1) \times(0, \infty), \\ u(x, t)=\frac{\partial u(x, t)}{\partial v}=0 & \text { on } \partial \Omega \times(0, \infty), \\ u(x, 0)=u_{0}(x), u_{t}(x, 0)=u_{1}(x) & \text { in } \Omega, \\ z(x, \rho, 0)=f_{0}(x,-\tau \rho) & \text { in } \Omega \times(0,1), \\ z(x, 0, t)=u_{t}(x, t) & \text { in } \Omega \times(0, \infty) .\end{cases}
$$

Theorem 1. Assume that (3.1) holds and $m(\cdot)$ satisfies (1.2), (2.1) and $p(\cdot)$ satisfies (2.1) and

$$
2 \leq p^{-} \leq p(x) \leq p^{+} \leq \frac{2(n-2)}{n-4} \quad \text { if } n>4 .
$$

Assume further that $\left(u_{0}, u_{1}\right) \in H_{0}^{2}(\Omega) \times L^{2}(\Omega), f_{0} \in L^{m(\cdot)}(\Omega \times(0,1))$ and $T>0$. Then, the problem (3.3) has a unique local solution

$$
\begin{aligned}
u & \in C\left([0, T] ; H_{0}^{2}(\Omega)\right), \\
u_{t} & \in C\left([0, T] ; L^{2}(\Omega)\right) \cap L^{m(\cdot)}(\Omega \times(0, T)), \\
z & \in L^{m(\cdot)}(\Omega \times(0,1)) .
\end{aligned}
$$


Proof. Existence: Let $v \in L^{\infty}\left((0, T) ; H_{0}^{2}(\Omega)\right)$. Since

$$
2\left(p^{-}-1\right) \leq 2\left(p^{+}-1\right) \leq \frac{2 n}{n-4},
$$

then

$$
\|h(v)\|^{2} \leq|b|^{2}\left\{\int_{\Omega}|v|^{2\left(p^{-}-1\right)} d x+\int_{\Omega}|v|^{2\left(p^{+}-1\right)} d x\right\}<\infty .
$$

Hence, we have

$$
h(v) \in L^{\infty}\left((0, T) ; L^{2}(\Omega)\right) \subset L^{2}(\Omega \times(0, T)) .
$$

Thus, for each $v \in L^{\infty}\left((0, T) ; H_{0}^{2}(\Omega)\right)$, there exists a unique solution

$$
\begin{aligned}
u & \in L^{\infty}\left((0, T) ; H_{0}^{2}(\Omega)\right), \\
u_{t} & \in L^{\infty}\left((0, T) ; L^{2}(\Omega)\right) \cap L^{m(\cdot)}(\Omega \times(0, T)), \\
z & \in L^{m(\cdot)}(\Omega \times(0,1))
\end{aligned}
$$

satisfying the following problem

$$
\begin{cases}u_{t t}+\Delta^{2} u-\Delta u_{t}+\mu_{1}\left|u_{t}(x, t)\right|^{m(x)-2} u_{t}(x, t) & \text { in } \Omega \times(0, T), \\ +\mu_{2}|z(x, 1, t)|^{m(x)-2} z(x, 1, t)=h(v) & \text { in } \Omega \times(0,1) \times(0, T), \\ \tau z_{t}(x, \rho, t)+z_{\rho}(x, \rho, t)=0 & \text { on } \partial \Omega \times(0, T), \\ u(x, t)=\frac{\partial u(x, t)}{\partial v}=0 & \text { in } \Omega, \\ u(x, 0)=u_{0}(x), u_{t}(x, 0)=u_{1}(x) & \text { in } \Omega \times(0, T), \\ z(x, 0, t)=u_{t}(x, t) & \text { in } \Omega \times(0,1) . \\ z(x, \rho, 0)=f_{0}(x,-\tau \rho) & \end{cases}
$$

Define the following space that the sequence $\left(u^{k}\right)$ is Cauchy in

$$
X:=C\left([0, T] ; H_{0}^{2}(\Omega)\right) \cap C^{1}\left([0, T] ; L^{2}(\Omega)\right),
$$

equipped with the norm

$$
\|u\|_{X}^{2}=\max _{0 \leq t \leq T}\left\{\left\|u_{t}\right\|^{2}+\|\Delta u\|^{2}\right\} .
$$

We define the nonlinear mapping $K: X \rightarrow X$ by $K(v)=u$, here, $u$ is the unique solution of (3.5). Now, we shall show that there exist $T>0$, such that

(i) $K: X \rightarrow X$,

(ii) $K$ is a contraction mapping in $X$. 
To show (i), we multiply the first equation in (3.5) by $u_{t}$ and integrate over $\Omega \times(0, t)$, to obtain

$$
\begin{aligned}
& \quad \frac{1}{2}\left\|u_{t}\right\|^{2}+\frac{1}{2}\|\Delta u\|^{2}+\int_{0}^{t}\left\|\nabla u_{t}\right\|^{2} d s+\mu_{1} \int_{0}^{t} \int_{\Omega}\left|u_{t}(s)\right|^{m(x)} d x d s \\
& +\mu_{2} \int_{0}^{t} \int_{\Omega}|z(x, 1, s)|^{m(x)-2} z(x, 1, s) u_{t}(s) d x d s \\
& =\frac{1}{2} \int_{\Omega} u_{1}^{2} d x+\frac{1}{2} \int_{\Omega}\left|\Delta u_{0}\right|^{2} d x+b \int_{0}^{t} \int_{\Omega}|v|^{p(x)-2} v u_{t}(s) d x d s .
\end{aligned}
$$

We multiply the second equation in (3.5) by $\frac{\zeta}{\tau} z^{m(x)-1}$, and integrate over $\Omega \times(0,1) \times(0, t)$, to have

$$
\begin{aligned}
\int_{0}^{1} \int_{\Omega} \frac{\zeta}{m(x)}\left(|z(x, \rho, t)|^{m(x)}-|z(x, \rho, 0)|^{m(x)}\right) d x d \rho & \\
& =\int_{0}^{t} \int_{\Omega} \frac{\zeta}{m(x) \tau}\left(|z(x, 0, s)|^{m(x)}-|z(x, 1, s)|^{m(x)}\right) d x d s .
\end{aligned}
$$

Combining (3.6) and (3.7), we have

$$
\begin{aligned}
& \frac{1}{2}\left\|u_{t}\right\|^{2}+\frac{1}{2}\|\Delta u\|^{2}+\int_{0}^{t}\left\|\nabla u_{t}\right\|^{2} d s+\int_{0}^{1} \int_{\Omega} \frac{\zeta}{m(x)}|z(x, \rho, t)|^{m(x)} d x d \rho \\
& +\mu_{1} \int_{0}^{t} \int_{\Omega}\left|u_{t}(s)\right|^{m(x)} d x d s+\mu_{2} \int_{0}^{t} \int_{\Omega}|z(x, 1, s)|^{m(x)-2} z(x, 1, s) u_{t}(s) d x d s \\
& +\int_{0}^{t} \int_{\Omega} \frac{\zeta}{m(x) \tau}\left(|z(x, 1, s)|^{m(x)}-\left|u_{t}(s)\right|^{m(x)}\right) d x d s \\
& =\frac{1}{2} \int_{\Omega} u_{1}^{2} d x+\frac{1}{2} \int_{\Omega}\left|\Delta u_{0}\right|^{2} d x+\int_{0}^{1} \int_{\Omega} \frac{\zeta}{m(x)}\left|f_{0}(x,-\tau \rho)\right|^{m(x)} d x d \rho \\
& +b \int_{0}^{t} \int_{\Omega}|v|^{p(x)-2} v u_{t}(s) d x d s
\end{aligned}
$$

Utilizing Young's inequality and (1.2), we get

$$
\begin{aligned}
& -\mu_{2} \int_{\Omega}|z(x, 1, s)|^{m(x)-2} z(x, 1, s) u_{t}(s) d x d s \\
& \quad \leq \frac{\left|\mu_{2}\right|}{m^{-}} \int_{\Omega}\left|u_{t}(s)\right|^{m(x)} d x+\frac{\left(m^{+}-1\right)\left|\mu_{2}\right|}{m^{+}} \int_{\Omega}|z(x, 1, s)|^{m(x)} d x .
\end{aligned}
$$

Applying Young's inequality and Sobolev embedding $H_{0}^{2}(\Omega) \hookrightarrow L^{\frac{2 n}{n-4}}(\Omega)$, we obtain

$$
\begin{aligned}
\left.\left|\int_{\Omega}\right| v\right|^{p(x)-2} v u_{t}(s) & \left.d x\left|\leq \frac{\varepsilon}{4} \int_{\Omega}\right| u_{t}(s)\right|^{2} d x+\frac{1}{\varepsilon} \int_{\Omega}|v|^{2(p(x)-1)} d x \\
\leq & \frac{\varepsilon}{4} \int_{\Omega}\left|u_{t}(s)\right|^{2} d x+\frac{c_{e}}{\varepsilon}\left\{\|\Delta v\|^{2\left(p^{-}-1\right)}+\|\Delta v\|^{2\left(p^{+}-1\right)}\right\},
\end{aligned}
$$


here, $c_{e}$ is the embedding constant. We should inserting (3.9) and (3.10) into (3.8), then, we have

$$
\begin{aligned}
& \frac{1}{2}\left\|u_{t}\right\|^{2}+\frac{1}{2}\|\Delta u\|^{2}+\int_{0}^{t}\left\|\nabla u_{t}\right\|^{2} d s+\int_{0}^{1} \int_{\Omega} \frac{\zeta}{m(x)}|z(x, \rho, t)|^{m(x)} d x d \rho \\
& +\left(\mu_{1}-\frac{\left|\mu_{2}\right|}{m^{-}}-\frac{\zeta}{m^{-} \tau}\right) \int_{0}^{t} \int_{\Omega}\left|u_{t}(s)\right|^{m(x)} d x d s \\
& +\left(\frac{\zeta}{m^{+} \tau}-\frac{\left(m^{+}-1\right)\left|\mu_{2}\right|}{m^{+}}\right) \int_{0}^{t} \int_{\Omega}|z(x, 1, s)|^{m(x)} d x d s \\
& \leq \frac{1}{2} \int_{\Omega} u_{1}^{2} d x+\frac{1}{2} \int_{\Omega}\left|\Delta u_{0}\right|^{2} d x+\int_{0}^{1} \int_{\Omega} \frac{\zeta}{m(x)}\left|f_{0}(x,-\tau \rho)\right|^{m(x)} d x d \rho \\
& +\frac{\varepsilon c T}{4} \sup _{(0, T)} \int_{\Omega}\left|u_{t}\right|^{2} d x+\frac{c_{e} c}{\varepsilon}\left\{\int_{0}^{T}\|\Delta v\|^{2\left(p^{-}-1\right)} d s+\int_{0}^{T}\|\Delta v\|^{2\left(p^{+}-1\right)} d s\right\} .
\end{aligned}
$$

By (3.2), we have

$$
\begin{aligned}
& \frac{1}{2} \sup _{(0, T)}\left\|u_{t}\right\|^{2}+\frac{1}{2} \sup _{(0, T)}\|\Delta u\|^{2}+\frac{\zeta}{m^{+}}\|z(x, \rho, t)\|_{L^{m(\cdot)}(\Omega \times(0,1))}^{m(\cdot)} \\
& \leq \frac{1}{2} \int_{\Omega} u_{1}^{2} d x+\frac{1}{2} \int_{\Omega}\left|\Delta u_{0}\right|^{2} d x+\frac{\zeta}{m^{-}} \int_{0}^{1} \int_{\Omega}\left|f_{0}(x,-\tau \rho)\right|^{m(x)} d x d \rho \\
&+\frac{\varepsilon c T}{4} \sup _{(0, T)}\left\|u_{t}\right\|^{2}+\frac{c_{e} c T}{\varepsilon}\left\{\|v\|_{X}^{2\left(p^{-}-1\right)}+\|v\|_{X}^{2\left(p^{+}-1\right)}\right\} .
\end{aligned}
$$

By taking $\varepsilon$ such that $\varepsilon c T=1$, we get

$$
\begin{aligned}
\|u\|_{X}^{2} \leq \frac{c^{*}}{2} \int_{\Omega} u_{1}^{2} d x+\frac{c^{*}}{2} \int_{\Omega}\left|\Delta u_{0}\right|^{2} d x+\frac{c^{*} \zeta}{m^{-}} \int_{0}^{1} \int_{\Omega}\left|f_{0}(x,-\tau \rho)\right|^{m(x)} d x d \rho \\
+c_{*} T\left\{\|v\|_{X}^{2\left(p^{-}-1\right)}+\|v\|_{X}^{2\left(p^{+}-1\right)}\right\},
\end{aligned}
$$

where $\frac{1}{c^{*}}=\min \left\{\frac{1}{4}, \frac{\zeta}{m^{+}}\right\}$and $c_{*}=\frac{c^{*} c_{e} c}{\varepsilon}$. Here, we choose $M>0$ large enough, such that $\|v\|_{X} \leq M$, then

$$
c^{*} \int_{\Omega} u_{1}^{2} d x+c^{*} \int_{\Omega}\left|\Delta u_{0}\right|^{2} d x+\frac{2 c^{*} \zeta}{m^{-}} \int_{0}^{1} \int_{\Omega}\left|f_{0}(x,-\tau \rho)\right|^{m(x)} d x d \rho \leq M^{2}
$$

and $T$ sufficiently small such that

$$
T \leq \frac{1}{2 c_{*}\left(M^{2\left(p^{-}-2\right)}+M^{2\left(p^{+}-2\right)}\right)} .
$$

As a result, we have

$$
\|u\|_{X}^{2} \leq M^{2}
$$


Thus we have $K: Z \rightarrow Z$, where

$$
Z=\left\{u \in X \text { such that }\|u\|_{X} \leq M\right\} .
$$

Next, we show that $K$ is a contraction mapping. For this purpose, we let $K\left(v^{1}\right)=u^{1}$ and $K\left(v^{2}\right)=u^{2}$ and set $u=u^{1}-u^{2}$ and $w=w^{1}-w^{2}$ then $u$ and $w$ satisfy

$$
\begin{cases}u_{t t}+\Delta^{2} u-\Delta u_{t}+\mu_{1}\left|u_{t}^{1}(x, t)\right|^{m(x)-2} u_{t}^{1}(x, t) & \\ -\mu_{1}\left|u_{t}^{2}(x, t)\right|^{m(x)-2} u_{t}^{2}(x, t) & \\ +\mu_{2}\left|z^{1}(x, 1, t)\right|^{m(x)-2} z^{1}(x, 1, t) & \text { in } \Omega \times(0, T), \\ -\mu_{2}\left|z^{2}(x, 1, t)\right|^{m(x)-2} z^{2}(x, 1, t) & \\ =b\left|v^{1}\right|^{p(x)-2} v^{1}-b\left|v^{2}\right|^{p(x)-2} v^{2} & \\ u(x, t)=\frac{\partial u(x, t)}{\partial v}=0 & \text { on } \partial \Omega \times(0, T), \\ z(x, 0, t)=u_{t}(x, t) & \text { in } \Omega \times(0, T), \\ z(x, \rho, 0)=0 & \text { in } \Omega \times(0,1), \\ u(x, 0)=0, u_{t}(x, 0)=0 & \text { in } \Omega .\end{cases}
$$

We multiply equation (3.11) by $u_{t}$ and integrate over $\Omega \times(0, t)$, we get

$$
\begin{aligned}
& \frac{1}{2}\left\|u_{t}\right\|^{2}+\frac{1}{2}\|\Delta u\|^{2}+\int_{0}^{t}\left\|\nabla u_{t}\right\|^{2} d s \\
&+\mu_{1} \int_{0}^{t} \int_{\Omega}\left(\left|u_{t}^{1}(s)\right|^{m(x)-2} u_{t}^{1}(s)-\left|u_{t}^{2}(s)\right|^{m(x)-2} u_{t}^{2}(s)\right) u_{t}(s) d x d s \\
&+\mu_{2} \int_{0}^{t} \int_{\Omega}\left(\left|z^{1}(x, 1, s)\right|^{m(x)-2} z^{1}(x, 1, s)-\left|z^{2}(x, 1, s)\right|^{m(x)-2} z^{2}(x, 1, s)\right) u_{t}(s) d x d s \\
& \quad=\int_{0}^{t} \int_{\Omega}\left(h\left(v_{1}\right)-h\left(v_{2}\right)\right) u_{t}(s) d x d s,
\end{aligned}
$$

where $h(v)=b|v|^{p(x)-2} v$. Since the function $u \rightarrow|u|^{m(x)-2} u$ is increasing, we conclude that

$$
\frac{1}{2}\left\|u_{t}\right\|^{2}+\frac{1}{2}\|\Delta u\|^{2}+\int_{0}^{t}\left\|\nabla u_{t}\right\|^{2} d s \leq \int_{0}^{t} \int_{\Omega}\left(h\left(v_{1}\right)-h\left(v_{2}\right)\right) u_{t}(s) d x d s .
$$

Thanks to (3.4), Young's inequality and Sobolev embedding, we obtain

$$
\begin{aligned}
& \int_{\Omega}\left|h\left(v_{1}\right)-h\left(v_{2}\right)\right|\left|u_{t}(s)\right| d x=\int_{\Omega}\left|h^{\prime}(\rho)\right|\|v\||| u_{t}(s) \mid d x \\
& \leq \frac{\delta_{0}}{2} \int_{\Omega}\left|u_{t}(s)\right|^{2} d x+\frac{1}{2 \delta_{0}} \int_{\Omega}\left|h^{\prime}(\rho)\right|^{2}|v|^{2} d x \leq \frac{\delta_{0}}{2} \int_{\Omega}\left|u_{t}(s)\right|^{2} d x \\
& +\frac{b^{2}\left(p^{+}-1\right)^{2}}{2 \delta_{0}}\left[\left(\int_{\Omega}|\rho|^{\frac{n\left(p^{-}-2\right)}{2}} d x\right)^{\frac{4}{n}}+\left(\int_{\Omega}|\rho|^{\frac{n\left(p^{+}-2\right)}{2}} d x\right)^{\frac{4}{n}}\right]\left(\int_{\Omega}|v|^{\frac{2 n}{n-4}} d x\right)^{\frac{n-4}{n}}
\end{aligned}
$$




$$
\begin{aligned}
& \leq \frac{\delta_{0}}{2}\left\|u_{t}(s)\right\|^{2}+\frac{b^{2}\left(p^{+}-1\right)^{2} c_{e}}{2 \delta_{0}}\left[\|\Delta \rho\|^{2\left(p^{-}-2\right)}+\|\Delta \rho\|^{2\left(p^{+}-2\right)}\right]\|\Delta v\|^{2} \\
& \leq \frac{\delta_{0}}{2}\left\|u_{t}(s)\right\|^{2}+\frac{b^{2}\left(p^{+}-1\right)^{2} c_{e}}{\delta_{0}}\left(M^{2\left(p^{-}-2\right)}+M^{2\left(p^{+}-2\right)}\right)\|\Delta v\|^{2},
\end{aligned}
$$

where $v=v_{1}-v_{2}$ and $\rho=\vartheta v_{1}+(1-\vartheta) v_{2}, 0 \leq \vartheta \leq 1$.

By inserting (3.13) into (3.12) and choosing $\delta_{0}$ small enough, we obtain

$$
\|u\|_{X}^{2} \leq d\|v\|_{X}^{2}
$$

where $d=\frac{4 b^{2}\left(p^{+}-1\right)^{2} c_{e} T}{\delta_{0}}\left(M^{2\left(p^{-}-2\right)}+M^{2\left(p^{+}-2\right)}\right)$.

Now, we choose $T$ small enough so that $0<d<1$. Thus, (3.14) indicates that $K$ is a contraction. The Banach fixed theorem shows that the existence of a unique $u \in Z$ satisfying $K(u)=u$. Obviously, it is a solution of (3.3).

Uniqueness: Assume that (3.3) have two solutions $\left(u^{1}, z^{1}\right),\left(u^{2}, z^{2}\right)$. We define $\tilde{u}=u^{1}-u^{2}$ and $\tilde{z}=z^{1}-z^{2}$, then $(\tilde{u}, \tilde{z})$ satisfy

$$
\begin{cases}\tilde{u}_{t t}+\Delta^{2} \tilde{u}-\Delta \tilde{u}_{t}+\mu_{1}\left|u_{t}^{1}(t)\right|^{m(x)-2} u_{t}^{1}(t) & \\ -\mu_{1}\left|u_{t}^{2}(t)\right|^{m(x)-2} u_{t}^{2}(x, t) & \\ +\mu_{2}\left|z^{1}(x, 1, t)\right|^{m(x)-2} z^{1}(x, 1, t) & \text { in } \Omega \times(0, T), \\ -\mu_{2}\left|z^{2}(x, 1, t)\right|^{m(x)-2} z^{2}(x, 1, t) & \\ =b u^{1}\left|u^{1}\right|^{p(x)-2}-b u^{2}\left|u^{2}\right|^{p(x)-2} & \\ \tau \widetilde{z}_{t}(x, \rho, t)+\widetilde{z_{\rho}}(x, \rho, t)=0 & \text { in } \Omega \times(0,1) \times(0, T), \\ \tilde{u}(x, t)=\frac{\partial \tilde{u}(x, t)}{\partial v}=0 & \text { in } \partial \Omega \times(0, T), \\ \widetilde{z}(x, 0, t)=\widetilde{u}_{t}(x, t) & \text { in } \Omega \times(0, T), \\ \tilde{z}(x, \rho, 0)=0 & \text { in } \Omega \times(0,1), \\ \tilde{u}(x, 0)=0 \tilde{u}_{t}(x, 0)=0 & \text { in } \Omega .\end{cases}
$$

We multiply the first equation in (3.15) by $\tilde{u}_{t}$ and integrate over $\Omega$, we get

$$
\begin{aligned}
& \frac{1}{2} \frac{d}{d t}\left\{\int_{\Omega}\left|\tilde{u}_{t}\right|^{2} d x+\int_{\Omega}|\Delta \tilde{u}|^{2} d x\right\}+\int_{\Omega}\left|\nabla \tilde{u}_{t}\right|^{2} d x \\
& +\mu_{1} \int_{\Omega}\left(\left|u_{t}^{1}(t)\right|^{m(x)-2} u_{t}^{1}(t)-\left|u_{t}^{2}(t)\right|^{m(x)-2} u_{t}^{2}(x, t)\right) \tilde{u}_{t}(t) d x \\
& +\mu_{2} \int_{\Omega}\left(\left|z^{1}(x, 1, t)\right|^{m(x)-2} z^{1}(x, 1, t)-\left|z^{2}(x, 1, t)\right|^{m(x)-2} z^{2}(x, 1, t)\right) \tilde{u}_{t}(t) d x \\
& =b \int_{\Omega}\left(u^{1}\left|u^{1}\right|^{p(x)-2}-b u^{2}\left|u^{2}\right|^{p(x)-2}\right) \tilde{u}_{t}(t) d x .
\end{aligned}
$$


Multiplying the second equation in (3.15) by $\tilde{z}$ and integrating over $\Omega \times(0,1)$, we have

$$
\frac{\tau}{2} \frac{d}{d t} \int_{0}^{1} \int_{\Omega}|\tilde{z}(x, \rho, t)|^{2} d x d \rho+\frac{1}{2}\left(\|\tilde{z}(x, 1, t)\|^{2}-\left\|\tilde{u}_{t}(t)\right\|^{2}\right)=0 .
$$

Combining (3.16) and (3.17), we have

$$
\begin{gathered}
\frac{1}{2} \frac{d}{d t}\left\{\int_{\Omega}\left|\tilde{u}_{t}(t)\right|^{2} d x+\int_{\Omega}|\tilde{\Delta u}(t)|^{2} d x+\left.\tau|| \tilde{z}(x, \rho, t)\right|_{L^{2}(\Omega \times(0,1))} ^{2}\right\}+\int_{\Omega}\left|\nabla \tilde{u}_{t}\right|^{2} d x \\
+\frac{1}{2}\|\tilde{z}(x, 1, t)\|^{2}+\mu_{1} \int_{\Omega}\left(\left|u_{t}^{1}(t)\right|^{m(x)-2} u_{t}^{1}(t)-\left|u_{t}^{2}(t)\right|^{m(x)-2} u_{t}^{2}(t)\right) \tilde{u}_{t}(t) d x \\
+\mu_{2} \int_{\Omega}\left(\left|z^{1}(x, 1, t)\right|^{m(x)-2} z^{1}(x, 1, t)-\left|z^{2}(x, 1, t)\right|^{m(x)-2} z^{2}(x, 1, t)\right) \tilde{u}_{t}(t) d x \\
=b \int_{\Omega}\left(u^{1}\left|u^{1}\right|^{p(x)-2}-b u^{2}\left|u^{2}\right|^{p(x)-2}\right) \tilde{u}_{t}(t) d x+\frac{1}{2}\left\|\tilde{u}_{t}(t)\right\|^{2}
\end{gathered}
$$

Since the function $y \rightarrow|y|^{m(\cdot)-2} y$ is increasing, we get

$$
\begin{array}{r}
\int_{\Omega}\left(\left|u_{t}^{1}(t)\right|^{m(x)-2} u_{t}^{1}(t)-\left|u_{t}^{2}(t)\right|^{m(x)-2} u_{t}^{2}(t)\right) \tilde{u}_{t}(t) d x \geq 0, \\
\int_{\Omega}\left(\left|z^{1}(x, 1, t)\right|^{m(x)-2} z^{1}(x, 1, t)-\left|z^{2}(x, 1, t)\right|^{m(x)-2} z^{2}(x, 1, t)\right) \tilde{u}_{t}(t) d x \geq 0 .
\end{array}
$$

By using (3.18), (3.19) and (3.20), we obtain

$$
\begin{array}{r}
\frac{1}{2} \frac{d}{d t}\left\{\int_{\Omega}\left\|\tilde{u}_{t}(t)\right\|^{2}+\|\Delta \tilde{u}(t)\|^{2}+\tau\|\tilde{z}(x, \rho, t)\|_{L^{2}(\Omega \times(0,1))}^{2}\right\}+\frac{1}{2}\|\tilde{z}(x, 1, t)\|^{2} \\
\leq c\left(\left\|\tilde{u}_{t}(t)\right\|^{2}+\|\Delta \tilde{u}(t)\|^{2}\right)
\end{array}
$$

which implies that $\tilde{u}=0, \tilde{z}=0$.

\section{BLOW UP}

In this part, for the case $b>0$, we establish the blow-up result for our main problem (1.1). Now we introduce, as in the work of [15], the new function

$$
z(x, \rho, t)=u_{t}(x, t-\tau \rho), \quad x \in \Omega, \quad \rho \in(0,1), \quad t>0,
$$

which implies that

$$
\tau z_{t}(x, \rho, t)+z_{\rho}(x, \rho, t)=0, \quad x \in \Omega, \quad \rho \in(0,1), \quad t>0 .
$$


Consequently, problem (1.1) is equivalent to:

$$
\begin{cases}u_{t t}+\Delta^{2} u-\Delta u_{t}+\mu_{1} u_{t}(x, t)\left|u_{t}(x, t)\right|^{m(x)-2} & \\ +\mu_{2} z(x, 1, t)|z(x, 1, t)|^{m(x)-2} & \text { in } \Omega \times(0, \infty), \\ =b u|u|^{p(x)-2} & \\ \tau z_{t}(x, \rho, t)+z_{\rho}(x, \rho, t)=0 & \text { in } \Omega \times(0,1) \times(0, \infty), \\ z(x, \rho, 0)=f_{0}(x,-\rho \tau) & \text { in } \Omega \times(0,1), \\ u(x, t)=\frac{\partial u(x, t)}{\partial v}=0 & \text { on } \partial \Omega \times[0, \infty), \\ u(x, 0)=u_{0}(x), u_{t}(x, 0)=u_{1}(x) & \text { in } \Omega .\end{cases}
$$

We define the energy functional of (4.1) as

$$
E(t)=\frac{1}{2}\left\|u_{t}\right\|^{2}+\frac{1}{2}\|\Delta u\|^{2}+\int_{0}^{1} \int_{\Omega} \frac{\xi(x)|z(x, \rho, t)|^{m(x)}}{m(x)} d x d \rho-b \int_{\Omega} \frac{|u|^{p(x)}}{p(x)} d x,
$$

for $t \geq 0$, where $\xi$ is a continuous function satisfies

$$
\tau\left|\mu_{2}\right|(m(x)-1)<\xi(x)<\tau\left(\mu_{1} m(x)-\left|\mu_{2}\right|\right), \quad x \in \bar{\Omega} .
$$

The following lemma gives that, under the condition $\mu_{1}>\left|\mu_{2}\right|, E(t)$ is nonincreasing.

Lemma 8. Let $(u, z)$ be a solution of (4.1). Then there exists some $C_{0}>0$ such that

$$
E^{\prime}(t) \leq-C_{0} \int_{\Omega}\left(\left|u_{t}\right|^{m(x)}+|z(x, 1, t)|^{m(x)}\right) d x \leq 0 .
$$

Proof. We multiply the first equation in (4.1) by $u_{t}$, integrate over $\Omega$, then multiplying the second equation of (4.1) by $\frac{1}{\tau} \xi(x)|z|^{m(x)-2} z$ and integrate over $\Omega \times(0,1)$, summing up, we get

$$
\begin{array}{r}
\frac{d}{d t}\left[\frac{1}{2}\left\|u_{t}\right\|^{2}+\frac{1}{2}\|\Delta u\|^{2}+\int_{0}^{1} \int_{\Omega} \frac{\xi(x)|z(x, \rho, t)|^{m(x)}}{m(x)} d x d \rho-b \int_{\Omega} \frac{|u|^{p(x)}}{p(x)} d x\right] \\
=-\left\|\nabla u_{t}\right\|^{2}-\mu_{1} \int_{\Omega}\left|u_{t}\right|^{m(x)} d x-\frac{1}{\tau} \int_{\Omega} \int_{0}^{1} \xi(x)|z(x, \rho, t)|^{m(x)-2} z z_{\rho}(x, \rho, t) d \rho d x \\
\quad-\mu_{2} \int_{\Omega} u_{t} z(x, 1, t)|z(x, 1, t)|^{m(x)-2} d x
\end{array}
$$

Next, we estimate the last two terms of the right-hand side of (4.3) as following,

$$
\begin{aligned}
-\frac{1}{\tau} \int_{\Omega} \int_{0}^{1} \xi(x) \mid & \left.z(x, \rho, t)\right|^{m(x)-2} z z_{\rho}(x, \rho, t) d \rho d x \\
& =-\frac{1}{\tau} \int_{\Omega} \int_{0}^{1} \frac{\partial}{\partial \rho}\left(\frac{\xi(x)|z(x, \rho, t)|^{m(x)}}{m(x)}\right) d \rho d x \\
= & \frac{1}{\tau} \int_{\Omega} \frac{\xi(x)}{m(x)}\left(|z(x, 0, t)|^{m(x)}-|z(x, 1, t)|^{m(x)}\right) d x
\end{aligned}
$$




$$
=\int_{\Omega} \frac{\xi(x)}{\tau m(x)}\left|u_{t}\right|^{m(x)} d x-\int_{\Omega} \frac{\xi(x)}{\tau m(x)}|z(x, 1, t)|^{m(x)} .
$$

Using the Young's inequality, $q=\frac{m(x)}{m(x)-1}$ and $q^{\prime}=m(x)$ for the last term to obtain

$$
\left|u_{t}\right||z(x, 1, t)|^{m(x)-1} \leq \frac{1}{m(x)}\left|u_{t}\right|^{m(x)}+\frac{m(x)-1}{m(x)}|z(x, 1, t)|^{m(x)} .
$$

Consequently, we deduce that

$$
\begin{aligned}
& -\mu_{2} \int_{\Omega} u_{t} z|z(x, 1, t)|^{m(x)-2} d x \\
& \quad \leq\left|\mu_{2}\right|\left(\int_{\Omega} \frac{1}{m(x)}\left|u_{t}(t)\right|^{m(x)} d x+\int_{\Omega} \frac{m(x)-1}{m(x)}|z(x, 1, t)|^{m(x)} d x\right) .
\end{aligned}
$$

So

$$
\begin{aligned}
\frac{d E(t)}{d t} \leq-\int_{\Omega}\left[\mu_{1}-\left(\frac{\xi(x)}{\tau m(x)}\right.\right. & \left.\left.+\frac{\left|\mu_{2}\right|}{m(x)}\right)\right]\left|u_{t}(t)\right|^{m(x)} d x \\
& -\int_{\Omega}\left(\frac{\xi(x)}{\tau m(x)}-\frac{\left|\mu_{2}\right|(m(x)-1)}{m(x)}\right)|z(x, 1, t)|^{m(x)} d x .
\end{aligned}
$$

As a result, for all $x \in \bar{\Omega}$, the relation (4.2) satisfies,

$$
\begin{aligned}
& f_{1}(x)=\mu_{1}-\left(\frac{\xi(x)}{\tau m(x)}+\frac{\left|\mu_{2}\right|}{m(x)}\right)>0, \\
& f_{2}(x)=\frac{\xi(x)}{\tau m(x)}-\frac{\left|\mu_{2}\right|(m(x)-1)}{m(x)}>0 .
\end{aligned}
$$

Since $m(x)$, and hence $\xi(x)$, is bounded, we infer that $f_{1}(x)$ and $f_{2}(x)$ are also bounded. So, if we define

$$
C_{0}(x)=\min \left\{f_{1}(x), f_{2}(x)\right\}>0 \text { for any } x \in \bar{\Omega},
$$

and take $C_{0}(x)=\inf _{\bar{\Omega}} C_{0}(x)$, so $C_{0}(x) \geq C_{0}>0$. Hence,

$$
E^{\prime}(t) \leq-C_{0}\left[\int_{\Omega}\left|u_{t}(t)\right|^{m(x)} d x+\int_{\Omega}|z(x, 1, t)|^{m(x)} d x\right] \leq 0 .
$$

To prove the blow-up result, we assume that $E(0)<0$ in addition to (1.2). Set $H(t)=-E(t)$, hence $H^{\prime}(t)=-E^{\prime}(t) \geq 0$,

$$
0<H(0) \leq H(t) \leq b \int_{\Omega} \frac{|u|^{p(x)}}{p(x)} d x \leq \frac{b}{p^{-}} \rho(u),
$$

where

$$
\rho(u)=\rho_{p(\cdot)}(u)=\int_{\Omega}|u|^{p(x)} d x .
$$


Lemma 9 ([6, Lemma 3.2]). Suppose that condition (1.2) satisfies.Then, depending on $\Omega$ only, there exists a positive $C>1$, such that

$$
\rho^{s / p^{-}}(u) \leq C\left(\|\Delta u\|^{2}+\rho(u)\right) .
$$

Then, we have following inequalities:

$$
\begin{aligned}
\|u\|_{p^{-}}^{s} & \leq C\left(\|\Delta u\|^{2}+\|u(t)\|_{p^{-}}^{p^{-}}\right) \\
\rho^{s / p^{-}}(u) & \leq C\left(|H(t)|+\left\|u_{t}\right\|^{2}+\rho(u)+\int_{0}^{1} \int_{\Omega} \frac{\xi(x)|z(x, \rho, t)|^{m(x)}}{m(x)} d x d \rho\right), \\
\|u\|_{p^{-}}^{s} & \leq C\left(|H(t)|+\left\|u_{t}\right\|^{2}+\|u\|_{p^{-}}^{p^{-}}+\int_{0}^{1} \int_{\Omega} \frac{\xi(x)|z(x, \rho, t)|^{m(x)}}{m(x)} d x d \rho\right),
\end{aligned}
$$

for any $u \in H_{0}^{1}(\Omega)$ and $2 \leq s \leq p^{-}$. Let $(u, z)$ be a solution of (4.1), then

$$
\rho(u) \geq C\|u\|_{p^{-}}^{p^{-}}, \quad \int_{\Omega}|u|^{m(x)} d x \leq C\left(\rho^{m^{-} / p^{-}}(u)+\rho^{m^{+} / p^{-}}(u)\right) .
$$

The blow-up result is given by the following theorem:

Theorem 2. Let conditions (1.2) and (2.1) be provided and assume that $E(0)<0$. Then, the solution (4.1) blows up in finite time $T^{*}$, and

$$
T^{*} \leq \frac{1-\alpha}{\Psi \alpha[L(0)]^{\alpha /(1-\alpha)}},
$$

where $L(t)$ and $\alpha$ are given in (4.6) and (4.7), respectively.

Proof. Define

$$
L(t)=H^{1-\alpha}(t)+\varepsilon \int_{\Omega} u u_{t} d x+\frac{\varepsilon}{2}\|\nabla u\|^{2},
$$

where $\varepsilon$ small to be chosen later and

$$
0 \leq \alpha \leq \min \left\{\frac{p^{-}-2}{2 p^{-}}, \frac{p^{-}-m^{-}}{p^{-}\left(m^{+}-1\right)}, \frac{p^{-}-m^{+}}{p^{-}\left(m^{+}-1\right)}\right\} .
$$

Differentiation $L(t)$ with respect to $t$, and using the first equation in (4.1), we obtain

$$
\begin{aligned}
& L^{\prime}(t)=(1-\alpha) H^{-\alpha}(t) H^{\prime}(t)+\varepsilon \int_{\Omega}\left[u_{t}^{2}-|\Delta u|^{2}\right] d x \\
& +\varepsilon b \int_{\Omega}|u|^{p(x)} d x-\varepsilon \mu_{1} \int_{\Omega} u u_{t}(x, t)\left|u_{t}(x, t)\right|^{m(x)-2} d x \\
& \quad-\varepsilon \mu_{2} \int_{\Omega} u z(x, 1, t)|z(x, 1, t)|^{m(x)-2} d x .
\end{aligned}
$$


By using the definition of the $H(t)$ and for $0<a<1$, such that

$$
\begin{aligned}
L^{\prime}(t) \geq & C_{0}(1-\alpha) H^{-\alpha}(t)\left[\int_{\Omega}\left|u_{t}\right|^{m(x)} d x+\int_{\Omega}|z(x, 1, t)|^{m(x)} d x\right] \\
& +\varepsilon\left((1-a) p^{-} H(t)+\frac{(1-a) p^{-}}{2}\left\|u_{t}\right\|^{2}+\frac{(1-a) p^{-}}{2}\|\Delta u\|^{2}\right) \\
& +\varepsilon(1-a) p^{-} \int_{0}^{1} \int_{\Omega} \frac{\xi(x)|z(x, \rho, t)|^{m(x)}}{m(x)} d x d \rho \\
& +\varepsilon \int_{\Omega}\left[u_{t}^{2}-|\Delta u|^{2}\right] d x+\varepsilon a b \int_{\Omega}|u|^{p(x)} d x \\
& -\varepsilon \mu_{1} \int_{\Omega} u u_{t}(x, t)\left|u_{t}(x, t)\right|^{m(x)-2} d x-\varepsilon \mu_{2} \int_{\Omega} u z(x, 1, t)|z(x, 1, t)|^{m(x)-2} d x .
\end{aligned}
$$

Hence

$$
\begin{aligned}
L^{\prime}(t) \geq & C_{0}(1-\alpha) H^{-\alpha}(t)\left[\int_{\Omega}\left|u_{t}\right|^{m(x)} d x+\int_{\Omega}|z(x, 1, t)|^{m(x)} d x\right] \\
& +\varepsilon(1-a) p^{-} H(t)+\varepsilon \frac{(1-a) p^{-}+2}{2}\left\|u_{t}\right\|^{2}+\varepsilon \frac{(1-a) p^{-}-2}{2}\|\Delta u\|^{2} \\
& +\varepsilon(1-a) p^{-} \int_{0}^{1} \int_{\Omega} \frac{\xi(x)|z(x, \rho, t)|^{m(x)}}{m(x)} d x d \rho+\varepsilon a b \rho(u) \\
& -\varepsilon \mu_{1} \int_{\Omega} u u_{t}(x, t)\left|u_{t}(x, t)\right|^{m(x)-2} d x-\varepsilon \mu_{2} \int_{\Omega} u z(x, 1, t)|z(x, 1, t)|^{m(x)-2} d x .
\end{aligned}
$$

Utilizing Young's inequality, we get

$$
\int_{\Omega}\left|u_{t}\right|^{m(x)-1}|u| d x \leq \frac{1}{m^{-}} \int_{\Omega} \delta^{m(x)}|u|^{m(x)} d x+\frac{m^{+}-1}{m^{+}} \int_{\Omega} \delta^{-\frac{m(x)}{m(x)-1}}\left|u_{t}\right|^{m(x)} d x
$$

and

$$
\begin{aligned}
\int_{\Omega}|z(x, 1, t)|^{m(x)-1} & |u| d x \\
& \leq \frac{1}{m^{+}} \int_{\Omega} \delta^{m(x)}|u|^{m(x)} d x+\frac{m^{+}-1}{m^{+}} \int_{\Omega} \delta^{-\frac{m(x)}{m(x)-1}}|z(x, 1, t)|^{m(x)} d x .
\end{aligned}
$$

As in [14], estimates (4.8) and (4.9) remain valid if $\delta$ is time-dependent. Let us choose $\delta$ so that

$$
\delta^{-\frac{m(x)}{m(x)-1}}=k H^{-\alpha}(t),
$$

where $k \geq 1$ is specified later, we obtain

$$
\begin{gathered}
\int_{\Omega} \delta^{-\frac{m(x)}{m(x)-1}}\left|u_{t}\right|^{m(x)} d x=k H^{-\alpha}(t) \int_{\Omega}\left|u_{t}\right|^{m(x)} d x, \\
\int_{\Omega} \delta^{-\frac{m(x)}{m(x)-1}}|z(x, 1, t)|^{m(x)} d x=k H^{-\alpha}(t)|z(x, 1, t)|^{m(x)} d x
\end{gathered}
$$


and

$$
\begin{aligned}
\int_{\Omega} \delta^{m(x)}|u|^{m(x)} d x=\int_{\Omega} k^{1-m(x)} H^{\alpha(m(x)-1)}(t)|u|^{m(x)} d x & \\
& \leq \int_{\Omega} k^{1-m^{-}} H^{\alpha\left(m^{+}-1\right)}(t) \int_{\Omega}|u|^{m(x)} d x .
\end{aligned}
$$

By using (4.5), we obtain

$$
\begin{aligned}
H^{\alpha\left(m^{+}-1\right)}(t) \int_{\Omega}|u|^{m(x)} & d x \\
& \leq C\left[(\rho(u))^{m^{-} / p^{-}+\alpha\left(m^{+}-1\right)}+(\rho(u))^{m^{+} / p^{-}+\alpha\left(m^{+}-1\right)}\right] .
\end{aligned}
$$

From (4.7), we deduce that

$$
s=m^{-}+\alpha p^{-}\left(m^{+}-1\right) \leq p^{-} \quad \text { and } \quad s=m^{+}+\alpha p^{-}\left(m^{+}-1\right) \leq p^{-} .
$$

Then, by using Lemma 9, satisfies

$$
H^{\alpha\left(m^{+}-1\right)}(t) \int_{\Omega}|u|^{m(x)} d x \leq C\left(\|\Delta u\|^{2}+\rho(u)\right) .
$$

Combining (4.8)-(4.14), we get

$$
\begin{aligned}
L^{\prime}(t) \geq & (1-\alpha) H^{-\alpha}(t)\left[C_{0}-\varepsilon\left(\frac{m^{+}-1}{m^{+}}\right) c k\right] \int_{\Omega}\left|u_{t}\right|^{m(x)} d x \\
& +(1-\alpha) H^{-\alpha}(t)\left[C_{0}-\varepsilon\left(\frac{m^{+}-1}{m^{+}}\right) c k\right] \int_{\Omega}|z(x, 1, t)|^{m(x)} d x \\
& +\varepsilon\left(\frac{\left(p^{-}-2\right)-a p^{-}}{2}-\frac{C}{m^{-} k^{1-m^{-}}}\right)\|\Delta u\|^{2} \\
& +\varepsilon(1-a) p^{-} H(t)+\varepsilon \frac{(1-a) p^{-}+2}{2}\left\|u_{t}\right\|^{2}+\varepsilon\left(a b-\frac{C}{m^{-} k^{1-m^{-}}}\right) \rho(u) \\
& +\varepsilon(1-a) p^{-} \int_{0}^{1} \int_{\Omega} \frac{\xi(x)|z(x, \rho, t)|^{m(x)}}{m(x)} d x d \rho .
\end{aligned}
$$

Let us choose $a$ small enough such that

$$
\frac{(1-a) p^{-}+2}{2}>0
$$

and $k$ large enough so that

$$
\frac{\left(p^{-}-2\right)-a p^{-}}{2}-\frac{C}{m^{-} k^{1-m^{-}}}>0 \quad \text { and } \quad a b-\frac{C}{m^{-} k^{1-m^{-}}}>0 .
$$

Once $k$ and $a$ are fixed, picking $\varepsilon$ small enough such that

$$
C_{0}-\varepsilon\left(\frac{m^{+}-1}{m^{+}}\right) c k>0, \quad C_{0}-\varepsilon\left(\frac{m^{+}-1}{m^{+}}\right) c k>0
$$


and

$$
L(0)=H^{1-\alpha}(0)+\varepsilon \int_{\Omega} u_{0} u_{1} d x+\frac{\varepsilon}{2}\left\|\nabla u_{0}\right\|^{2}>0 .
$$

Consequently, (4.15) yields

$$
L^{\prime}(t) \geq \varepsilon \eta\left[H(t)+\left\|u_{t}\right\|^{2}+\|\Delta u\|^{2}+\rho(u)+\int_{0}^{1} \int_{\Omega} \frac{\xi(x)|z(x, \rho, t)|^{m(x)}}{m(x)} d x d \rho\right]
$$

for a constant $\eta>0$. Thus we get $L(t) \geq L(0)>0, \forall t \geq 0$.

Now, for some constants $\sigma, \Gamma>0$ we denote $L^{\prime}(t) \geq \Gamma L^{\sigma}(t)$. On the other hand, applying Hölder inequality, we obtain

$$
\left|\int_{\Omega} u u_{t} d x\right|^{1 /(1-\alpha)} \leq C\|u\|_{p^{-}}^{1 /(1-\alpha)}\left\|u_{t}\right\|_{2}^{1 /(1-\alpha)},
$$

and by using Young's inequality gives

$$
\left|\int_{\Omega} u u_{t} d x\right|^{1 /(1-\alpha)} \leq C\left[\|u\|_{p^{-}}^{m u /(1-\alpha)}+\left\|u_{t}\right\|_{2}^{\Theta /(1-\alpha)}\right]
$$

where $1 / \mu+1 / \Theta=1$. From (4.7), the choice of $\Theta=2(1-\alpha)$ will make $\mu /(1-\alpha)=2 /(1-2 \alpha) \leq p^{-}$. Hence,

$$
\left|\int_{\Omega} u u_{t} d x\right|^{1 /(1-\alpha)} \leq C\left[\|u\|_{p^{-}}^{s}+\left\|u_{t}\right\|^{2}\right]
$$

where $s=\mu /(1-\alpha)$. From (4.4), we have

$$
\left|\int_{\Omega} u u_{t} d x\right|^{1 /(1-\alpha)} \leq C\left[|H(t)|+\left\|u_{t}\right\|^{2}+\rho(u)+\int_{0}^{1} \int_{\Omega} \frac{\xi(x)|z(x, \rho, t)|^{m(x)}}{m(x)} d x d \rho\right] .
$$

Hence, we get

$$
\begin{gathered}
L^{1 /(1-\alpha)}(t)=\left[H^{(1-\alpha)}(t)+\varepsilon \int_{\Omega} u u_{t} d x+\frac{\varepsilon}{2}\|\nabla u\|^{2}\right]^{1 /(1-\alpha)} \\
\leq 2^{\alpha /(1-\alpha)}\left[H(t)+\left|\int_{\Omega} u u_{t} d x\right|^{1 /(1-\alpha)}\right] \\
\leq C\left[|H(t)|+\left\|u_{t}\right\|^{2}+\|\Delta u\|^{2}+\rho(u)+\int_{0}^{1} \int_{\Omega} \frac{\xi(x)|z(x, \rho, t)|^{m(x)}}{m(x)} d x d \rho\right]
\end{gathered}
$$

So, for some $\Psi>0$, from (4.16) we arrive

$$
L^{\prime}(t) \geq \Psi L^{1 /(1-\alpha)}(t)
$$

A simple integration of (4.17) over $(0, t)$ satisfies

$$
L^{\alpha /(1-\alpha)}(t) \geq \frac{1}{L^{-\alpha /(1-\alpha)}(0)-\Psi \alpha t /(1-\alpha)},
$$


which implies that the solution blows up in a finite time $T^{*}$, with

$$
T^{*} \leq \frac{1-\alpha}{\Psi \alpha[L(0)]^{\alpha /(1-\alpha)}} .
$$

As a result, the proof is completed.

\section{CONCLUSiONS}

In recent years, there has been published much work concerning the wave equation with constant delay or time-varying delay. However, to the best of our knowledge, there was no blow-up result for the Petrovsky equation with delay term and variable exponents. Firstly, we have been obtained the local existence result by using the Faedo-Galerkin method. Later, we have been proved that blow-up of solutions for problem (1.1) under the sufficient conditions in a bounded domain.

\section{REFERENCES}

[1] S. Antontsev, "Wave equation with $\mathrm{p}(\mathrm{x}, \mathrm{t})$-laplacian and damping term: existence and blow-up," Differ. Equ. Appl, vol. 3, no. 4, pp. 503-525, 2011.

[2] S. Antontsev, "Wave equation with p (x,t)-laplacian and damping term: Blow-up of solutions," Comptes Rendus Mécanique, vol. 339, no. 12, pp. 751-755, 2011, doi: 10.1016/j.crme.2011.09.001.

[3] Y. Chen, S. Levine, and M. Rao, "Variable exponent, linear growth functionals in image restoration," SIAM journal on Applied Mathematics, vol. 66, no. 4, pp. 1383-1406, 2006, doi: $10.1137 / 050624522$.

[4] L. Diening, P. Harjulehto, P. Hästö, and M. Růžička, Lebesgue and Sobolev spaces with variable exponents. Springer, 2011. doi: 10.1007/978-3-642-18363-8.

[5] X. Fan, J. Shen, and D. Zhao, "Sobolev embedding theorems for spaces wk, p $(x)(\Omega)$," Journal of Mathematical Analysis and Applications, vol. 262, no. 2, pp. 749-760, 2001, doi: 10.1006/jmaa.2001.7618.

[6] M. Kafini and S. Messaoudi, "On the decay and global nonexistence of solutions to a damped wave equation with variable-exponent nonlinearity and delay," in Annales Polonici Mathematici, vol. 122, doi: 10.4064/ap180524-31-10. Instytut Matematyczny Polskiej Akademii Nauk, 2019, pp. 49-70.

[7] M. Kafini and S. A. Messaoudi, "A blow-up result in a nonlinear wave equation with delay," Mediterranean Journal of Mathematics, vol. 13, no. 1, pp. 237-247, 2016, doi: 10.1007/s00009014-0500-4.

[8] J.-R. Kang, "Global nonexistence of solutions for von karman equations with variable exponents," Applied Mathematics Letters, vol. 86, pp. 249-255, 2018, doi: 10.1016/j.aml.2018.07.008.

[9] J.-R. Kang, "Existence and blow-up of solutions for von karman equations with time delay and variable exponents," Applied Mathematics and Computation, vol. 371, p. 124917, 2020, doi: 10.1016/j.amc.2019.124917.

[10] O. Kováčik and J. Rákosník, “On spaces $l^{p(x)}(\omega)$, and $w^{k, p(x)}(\omega)$," Czechoslovak mathematical journal, vol. 41, no. 4, pp. 592-618, 1991, doi: 0.21136/CMJ.1991.102493.

[11] G. Li, Y. Sun, and W. Liu, "Global existence and blow-up of solutions for a strongly damped petrovsky system with nonlinear damping," Applicable Analysis, vol. 91, no. 3, pp. 575-586, 2012, doi: 10.1080/00036811.2010.550576. 
[12] S. A. Messaoudi, "Global existence and nonexistence in a system of petrovsky," Journal of Mathematical Analysis and Applications, vol. 265, no. 2, pp. 296-308, 2002, doi: 10.1006/jmaa.2001.7697.

[13] S. A. Messaoudi and A. A. Talahmeh, "A blow-up result for a nonlinear wave equation with variable-exponent nonlinearities," Applicable Analysis, vol. 96, no. 9, pp. 1509-1515, 2017, doi: 10.1080/00036811.2016.1276170.

[14] S. A. Messaoudi, A. A. Talahmeh, and J. H. Al-Smail, "Nonlinear damped wave equation: Existence and blow-up," Computers \& Mathematics with Applications, vol. 74, no. 12, pp. 3024-3041, 2017, doi: 10.1016/j.camwa.2017.07.048.

[15] S. Nicaise and C. Pignotti, "Stability and instability results of the wave equation with a delay term in the boundary or internal feedbacks," SIAM Journal on Control and Optimization, vol. 45, no. 5, pp. 1561-1585, 2006, doi: 10.1137/060648891.

[16] S.-H. Park and J.-R. Kang, "Blow-up of solutions for a viscoelastic wave equation with variable exponents," Mathematical Methods in the Applied Sciences, vol. 42, no. 6, pp. 2083-2097, 2019.

[17] E. Pişkin, Sobolev Spaces. Seçkin Publishing, 2017.

[18] E. Pişkin, "Finite time blow up of solutions of the kirchhoff-type equation with variable exponents," International Journal of Nonlinear Analysis and Applications, vol. 11, no. 1, pp. 37-45, 2020, doi: 10.22075/ijnaa.2019.16022.1841.

[19] E. Pişkin and N. Polat, "On the decay of solutions for a nonlinear petrovsky equation," Mathematical Sciences Letters, vol. 3, no. 1, p. 43, 2014, doi: 10.12785/ms1/030107.

[20] M. Ragusa, "Regularity of solutions of divergence form elliptic equations," Proceedings of the American Mathematical Society, vol. 128, no. 2, pp. 533-540, 2000.

[21] M. A. Ragusa and A. Scapellato, "Mixed morrey spaces and their applications to partial differential equations," Nonlinear Analysis: Theory, Methods \& Applications, vol. 151, pp. 51-65, 2017, doi: 10.1016/j.na.2016.11.017.

[22] M. A. Ragusa and A. Tachikawa, "Regularity for minimizers for functionals of double phase with variable exponents," Advances in Nonlinear Analysis, vol. 9, no. 1, pp. 710-728, 2019, doi: 10.1515/anona-2020-0022.

[23] M. Růžička, Electrorheological fluids: modeling and mathematical theory. Springer Science \& Business Media, 2000. doi: 10.1007/BFb0104030.

[24] M. Shahrouzi, "On behaviour of solutions for a nonlinear viscoelastic equation with variableexponent nonlinearities," Computers \& Mathematics with Applications, vol. 75, no. 11, pp. 39463956, 2018, doi: 10.1016/j.camwa.2018.03.005.

[25] S.-T. Wu, L.-Y. Tsai et al., "On global solutions and blow-up of solutions for a nonlinearly damped petrovsky system," Taiwanese Journal of Mathematics, vol. 13, no. 2A, pp. 545-558, 2009, doi: 10.11650/twjm/1500405355.

Authors' addresses

Erhan Pişkin

Dicle University, Department of Mathematics, 21280 Diyarbakir, Turkey

E-mail address: episkin@dicle.edu.tr

Hazal Yüksekkaya

(Corresponding author) Dicle University, Department of Mathematics, 21280 Diyarbakir, Turkey

E-mail address: hazally.kayaegmail.com 